Of the 2,000 individual transformants obtained, about $10 \%$ showed a growth defect, and from those only $40 \%$ grew more slowly in the presence of galactose. A PCRbased procedure allowed the authors to amplify each DNA insert causing the galactose-dependent growth defect. Comparison of the sequences of the PCR products with public and proprietary C. albicans genome sequence databases gave some surprises. Among 86 identified genes, half were of unknown function, $75 \%$ of which (33 genes) had no homologs in genome databases of other organisms.

As expected, many of the genes isolated with known functions are essential in $S$. cerevisiae and other organisms, suggesting that the approach succeeded in identifying genes critical for the growth of C. albicans. To validate this hypothesis, De Backer et al. constructed heterozygote mutants of six randomly chosen genes among those identified (TEF3, TUF1, RPL27, RHO1, FAL1, and one gene encoding for a hypothetical protein, HYP1). Four heterozygotes showed a clear reduction of growth, a very uncommon feature when random heterozygotes are produced in C. albicans, thus indicating that genes critical for growth had been targeted.

With the engineered strains obtained in the initial screening test (so called "crippled" strains), De Backer et al. then performed high-throughput screening to identify candidate antifungal compounds. The rationale was to perform parallel growth assays of crippled strains with the wild-type strain in the presence of compound libraries. Positive compounds were identified as those enhancing the susceptibility of only the crippled strains in the presence of galactose. This selection is based on the assumption that the underexpression of components of an essential process leads to increased susceptibility to inhibitors of relevant steps in that process ${ }^{7}$. Using this small set of crippled strains with defects in known genes, the authors clearly demonstrated that specific compounds could specifically enhance the susceptibility of each individual strain, presumably by targeting the product of each selected gene.

It is interesting to note that a considerable number (33) of C. albicans essential genes (i.e., $38 \%$ of the total identified genes) had no known homologs among sequenced genomes. This suggests that the S. cerevisiae genome may not be the optimal source of basic information for the selection of candidate essential targets, as several pathogenspecific genes appear to be missing. On the other hand, several essential genes may not have been detected in this work because of the limited diversity of the antisense libraries

and the absence of integrations at specific gene loci.

In conclusion, the work of De Backer et al. is a nice example of how genome information, intelligent design of molecular tools,

\section{The work of De Backer et al. is a nice example of how genome information, intelligent design of molecular tools, and conventional drug screening on the relevant yeast pathogen can be combined.}

and conventional drug screening on the relevant yeast pathogen can be combined. The paper also provides geneticists with an approach to control, on a genome-wide scale, the expression of genes in C. albicans and assess their essentiality or their role in specific phenotypes, for example those asso- ciated with dimorphism (i.e. the ability to change cell morphology) or drug resistance.

The strategy is thus useful for identifying potential antifungal target genes with or without prior knowledge of sequence. Once the C. albicans genome has been completed, further selection of target genes can be made on the basis of their potential cellular role, the location of their translated products (e.g., localization in the membrane or cytosol), or their lack of equivalence in mammalian or human genomes. Finally, the strategy outlined here could be implemented in other diploid organisms with no sexual cycle.

1. De Backer, M. D. et al. Nat. Biotechnol. 19, 235-241 (2001).

2. Beck-Sague, C.M. \& Jarwis, W.R. J. Infect. Dis. 167 1247-1251 (1993)

3. Chiou, C.C., Groll, A.H. \& Walsh, T.J. Oncologist 5 , 120-135 (2000)

4. Georgopapadakou, N.H. Curr. Opin. Microbiol 1, 547-557 (1998)

5. Sanglard, D. et al. Antimicrob. Agents Chemother 39, 2378-2386 (1995).

6. Clarke, M.L., Patrikakis, M. \& Atkins, D. Biochem. Biophys. Res. Commun. 268, 8-13 (2000)

7. Giaever, G. et al. Nat. Genet. 21, 278-283 (1999).

\title{
Getting sense and finding function in protozoa
}

\section{Antisense using peptide nucleic acid promises to unlock the genetic secrets of Entamoeba, a major human pathogen.}

\section{Girija Ramakrishnan and William Petri}

Over the years, biologists have developed a whole battery of techniques for the routine and rapid genetic analysis of traditional model organisms, such as Escherichia coli, yeast, worms, and flies. Researchers working on more exotic organisms, however, are not so fortunate. Often, the methods available for dissecting gene function in these species are unreliable, labor-intensive, or both, and the complex and poorly characterized biology of the organism significantly stymies analysis. In this issue, Stock et $a l^{1}$. report use of antisense peptide nucleic acid (PNA) for the specific inhibition of genes in the protozoan pathogen, Entamoeba histolytica. The availability of a technology for rapidly assessing gene function in E. histolytica

Girija Ramakrishnan is research associate and William Petri is professor, the department of internal medicine, microbiology, and pathology, University of Virginia HSC, Charlottesville, VA 22908

(gr6q@virginia.edu). promises to facilitate research on an important contributor to acute and chronic diarrheal illness (amebiasis) worldwide.

Protozoan parasites occupy a unique niche in the biosphere because they are adapted to thrive at the expense of a living host. The diseases they cause impose both a heavy toll on human health-amebiasis

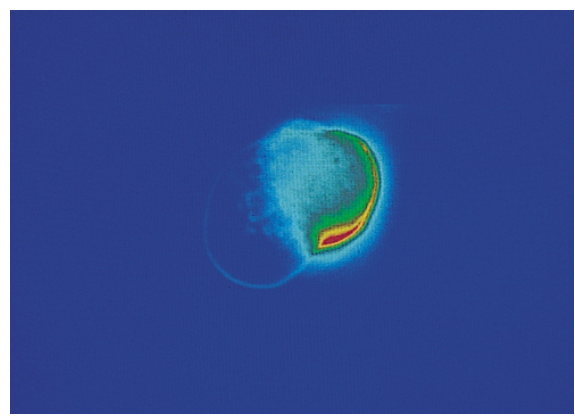

Figure 1. Epifluorescence phase contrast micrograph of $E$. histolytica with capped cellsurface lectin. Genetic analysis of the this organism will be facilitated by the work of Stock et al. 
Table 1. Molecular techniques used to determine gene function in parasitic protozoa Technique Application

Genomics

Sequence homologies

All protozoa

Gene knockout

Chemical mutagenesis

Transposon mutagenesis

Gene replacement by homologous

recombination

Transcription knockout

Antisense through stable expression of E. histolytica

antisense constructs

Antisense in conjunction with

hammerhead ribozyme

PNA

RNA interference (RNAi)

Giardia lamblia

E. histolytica ${ }^{1}$

Trypanosoma brucei

Complementation of heterologous

mutants, including bacteria and yeast

Kinetoplastids, apicomplexans, and E. histolytica

Expression of proteins from

stable constructs

Overexpression

Dominant-negative mutant proteins

Epitope-tagged and green fluorescent

protein-fusion proteins

Kinetoplastids, apicomplexans, and E. histolytica

Kinetoplastids and E. histolytica

Kinetoplastids, apicomplexans,

and E. histolytica

Identification of stage-specific genes

Suppression subtractive hybridization

Gene arrays

Plasmodium berghei

Plasmodium falciparum and Toxoplasma gondif

Proteomics

Approach for identifying proteins in

complexes (e.g., two-dimensional electrophoresis and mass spectrometry)

Yeast one/two-hybrid systems for

identifying DNA-protein/protein-protein

interactions

Groups of parasites are referred to where the technique applies to many members of the group.

aKinetoplastids represent Trypanosoma and Leishmania spp.

${ }^{b}$ Apicomplexans refer to Toxoplasma gondii and Plasmodium spp.

CL.W. Bergman, personal communication.

alone is estimated to kill 100,000 people a year ${ }^{2}$ - and an economic burden on agriculture through the infection of livestock. In the past decade, the development of several molecular genetic methods for studying protozoan parasites (Table 1) has enabled significant advances in our understanding of parasite biology and therefore disease control.

It is important to remember that protozoan parasites are truly a very diverse group of organisms with many different adaptations for succeeding in varied host environments. Many of them have multiple life stages that are dependent on the host, and some require multiple hosts. Moreover, many protozoa have haploid genomes, others are diploid, some are haploid only over part of their life cycle, and yet others have poorly defined ploidies. Each parasite thus requires a set of genetic tools to be specifically developed because of its unique biology.
Entamoeba histolytica, the causative agent of amebiasis, is a case in point. Currently, no techniques for the chromosomal manipulation of this parasite are available, and all existing attempts to manipulate the genetics of this organism have focused on stable episomal transfection of either dominant mutant gene constructs or antisense constructs.

In the present paper, Stock et al ${ }^{1}$. use antisense PNA oligomers for the specific inhibition of genes in E. histolytica. PNA oligomers have a polyamide backbone in place of the sugar-phosphate DNA backbone and are uncharged and stable to cleavage by enzymes. The authors show that E. histolytica trophozoites readily take up the oligomers, which then specifically block expression either of an episomal neomycin resistance gene or of a chromosomal erd2 homolog.

As with any antisense approach, the inhibitory effects were partial, but in both instances, an effect on cell growth was observed. Although the utility of the technique remains to be proven using other genes, the current data certainly suggest that the method should facilitate rapid functional analysis of genes in this organism. This is particularly important because the E. histolytica genome project is currently well underway. (The combined efforts of The Institute for Genomics Research and the Sanger Centre promise a complete genome sequence shortly.)

In fact, genome projects are in progress for a host of protozoan parasites, including those responsible for malaria (Plasmodium spp.), sleeping sickness (Trypanosoma brucei), leishmaniasis (Leishmania major), and cryptosporidiosis (Cryptosporidium parvum). Additionally, large expressed sequence tag (EST) databases have been

\section{Sequence information from the protozoa genome projects, used in conjunction with molecular techniques, promises to facilitate identification of genes that determine pathogenicity.}

established for such parasites as the toxoplasmosis agent, Toxoplasma gondii, and the cause of Chagas disease, Trypanosoma cruzi.

Sequence information from these projects, used in conjunction with molecular techniques (see Table 1), promises to facilitate identification of genes that determine pathogenicity. Genes associated with pathogenicity that encode functions unique to a parasite may serve as potential candidates for diagnosis, therapy, and vaccine development. In addition, sequence comparisons between related organisms may also reveal putative drug targets.

Apart from Entamoeba, molecular genetics approaches are also being used to unravel the biology of kinetoplastid parasites, such as the diploid Leishmania ${ }^{3}$ and Trypanosoma spp., and of apicomplexan parasites, such as Plasmodium spp. and Toxoplasma gondii. The tremendous potential of DNA chip technology in identifying genes that are expressed during specific developmental stages of the life cycle has already been demonstrated in Plasmodium falciparum $^{4,5}$ and in T. gondii (J.C. Boothroyd, personal communication). Such arrays promise to provide much information regarding parasite biology and disease pathogenesis in the future.

In light of the rapidly accumulating sequence information from the various par- 
asite genome projects, there is an urgent need for molecular techniques that can replace traditional time-consuming chromosomal gene "knockout" methodologies and enable rapid screening of identified open reading frames (ORFs) for functional significance. One promising approach is that of RNA interference (RNAi), which has been used to systematically analyze predicted ORFs on two different chromosomes of Caenorhabditis.elegans ${ }^{6,7}$. RNAi has been shown to work for several genes in T. brucei $i^{8,9}$ and will likely prove useful for analysis of other genes in other protozoan parasites. Antisense PNA oligomer technology, as demonstrated by Stock et al., may also be an effective strategy for rapid functional analysis of parasite genes. With protozoan disease and drug resistance an ever-present global problem, the more technologies we have at our disposal for unraveling parasite biology, the better.

1. Stock, R.J. et al. Nat. Biotechnol. 19, 231-234 (2001).

2. WHO/PAHO/UNESCO. Report of a consultation of experts on amoebiasis. Weekly Epidemiological Report of the WHO 72, Vol. 14, pp. 97-99 (WHO, Geneva; 1997).

3. Spath, G.F. et al. Proc. Natl. Acad. Sci. USA 97 9258-9263 (2000)

4. Hayward, R.E. et al. Mol. Microbiol. 35, 6-14 (2000)

5. Mamoun, C.B. et al. Mol. Microbiol. 39, 26-36 (2001).

6. Fraser, A.G. et al. Nature 408, 325-330 (2000)

7. Gonczy, P. et al. Nature 408, 331-336 (2000)

8. Ngo, H. et al. Proc. Natl. Acad. Sci. USA 95, 14687-14692 (1998).

9. LaCount, D.J. et al. Mol. Biochem. Parasitol. 111, 67-76 (2000).

10. Gilchrist, E. A. et al. J. Biol. Chem. (2001), in press.

\section{A rapid coming of age in tree biotechnology}

\section{Introduction of two genes from Arabidopsis significantly accelerates the maturation of citrus trees.}

\section{Marcos Egea-Cortines and Julia Weiss}

Trees are characterized by an extended adolescence. In fact, the juvenile phase in certain species sometimes can last over 20 years. This is particularly important for commercial fruit/nut tree growers and breeders, because prolonged juvenile periods delay harvesting and the evaluation/breeding of new strains. In this issue, Martiñez-Zapater et $a l^{1}$. exploit existing knowledge of the genetic control of flower development in Arabidopsis to engineer orange trees that reach sexual maturity at least four years earlier than the wild type. Their results could have significant economic and scientific implications for the tree fruit industry.

The breeding of new strains of fruit/nut trees is tremendously important both in economic terms and for human nutrition. Until now, however, our ability to manipulate fruit/nut tree strains through genetics has been limited by the extended maturation period of these plants. In human terms, this has meant that a fruit tree breeder might have to convince his/her grandchildren to help analyze (or harvest) work started decades before. As an example, seeds from almond trees selected for self-compatibility

Marcos Egea-Cortines and Julia Weiss are at the Área de Genética, Escuela Técnica Superior de Ingenieros Agrónomos, Alfonso XIII 48, Universidad Politécnica de Cartagena, 30203 Cartagena, Spain (marcos.egea@upct.es) in Murcia (Spain) in 1972, were available for crossing only in 1989 and released as new varieties 10 years later after evaluation of the F1 generation (L. Egea, personal communication). During the juvenile period, it is not possible to measure yield capacity, flowering time, mature tree architecture, branching, or resistance. Trait analysis often requires between three and five years after the first flowers appear, when trees become fully productive ${ }^{2}$. As a result, tree breeding research is among the slowest moving fields in plant biotechnology.

Now MartiñezZapater et $a l^{1}$. have shown that basic knowledge obtained in the plant model system Arabidopsis can be exploited to change and maybe to create a whole new field in plant biotechnology. Using transgenic approaches, they demonstrate that the Arabidopsis genes APETALA 1 (AP1) and $L E A F Y(L F Y)$ complementary DNAs, under the control of the commonly used cauliflower mosaic virus $35 \mathrm{~S}$ promoter, can significantly alter the juvenile period of orange trees. As expected for normal orange trees, transgenic control plants flower in five or six years. In contrast, transgenic trees expressing either $L F Y$ or $A P 1$ show no such delay and flower the spring following transformation. This accelerated juvenile period (characterized by decreased thorn production and leaf shape) is heritable in crosses with nontransformed plants and in plants raised from self-pollination. In all cases, plants harboring either 35S:LFY or 35S:AP1 flower within a year after germination. Notably, plants expressing the AP1 gene always formed flowers at the proper time of the year (spring).

The work clearly demonstrates that the juvenile phase in trees can be manipulated by floral meristem identity genes ${ }^{3,4}$ from model organisms like Antirrhinum or Arabidopsis. To date, Squamosa (SQUA)/AP1 $1^{5,6}$ orthologs have been cloned in several plants and ectopic expression of AP1 in Arabidopsis shown to induce early flowering ${ }^{7}$. The work of Martiñez-Zapater $e t$ al. confirms these findings, demonstrating that floral primordia are produced sooner than expected in $A P 1 / L F Y$ transgenic plants, even though environmental control of the process imposes a flowering period coinci-

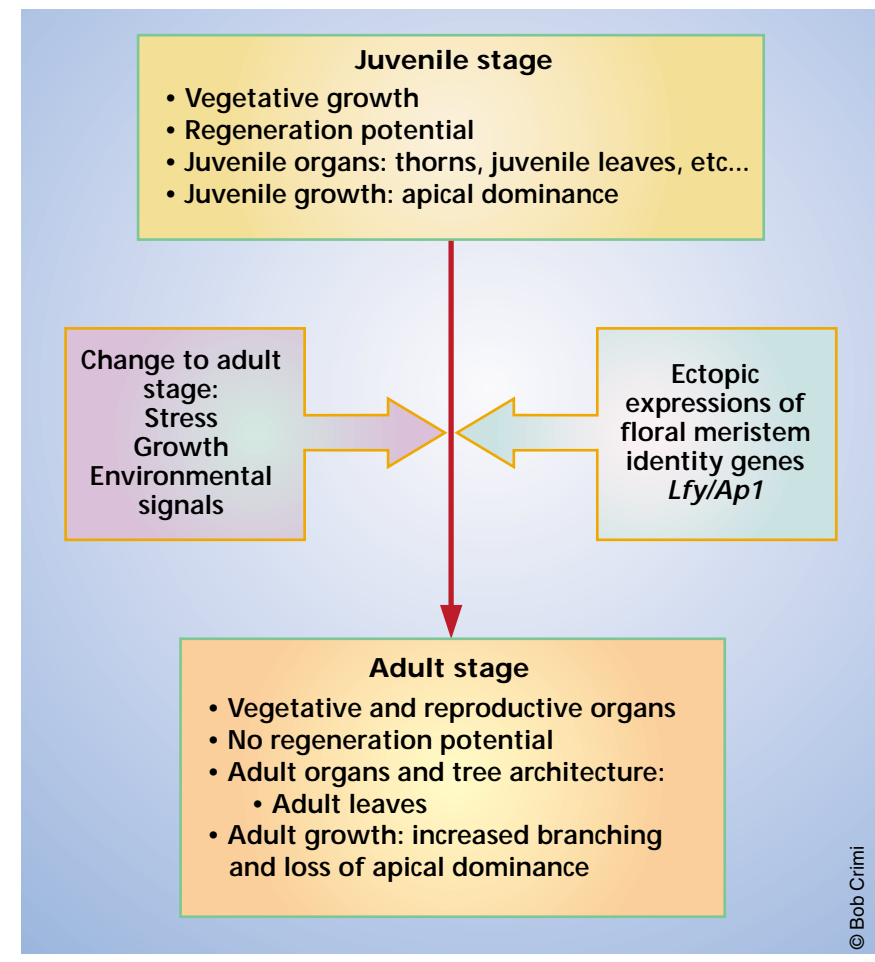

Figure 1. Reaching the next stage. Martiñez-Zapater have shown that ectopic expression of $L F Y / A P 1$ can significantly shorten the juvenile phase in the development of orange trees. 